\title{
Análise da variação qualitativa em amostras pequenas
}

\author{
F. G. BRIEGER \\ da Cadeira de Genética da \\ Inscola Superior de Agricultura \\ "Luiz de Queiroz"
}

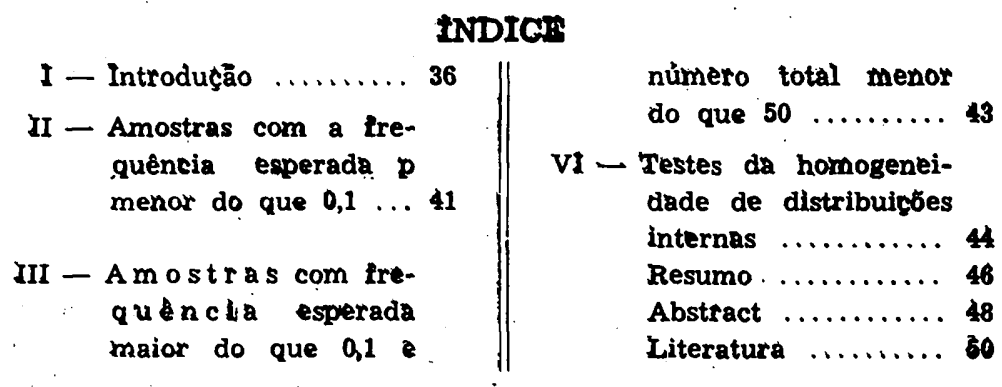

Apresentado para publicaçåo em 4-11-1047. 


\section{I - INTRODUÇAO}

Discuti num trabalho recente (BRIEGER, 1947) as métodos estatísticos que devemos empregar para determinar qual o número mínimo necessário em amostras para garantir o aparecimento de determinados tipos. $O$ objeto da presente publicaçá tem por fim explicar quais os processos de and́lise estatística que podem ser aplicados quando dispmos, por qualquer razăo, apenas de amostras muito pequenas.

A and́lise da variaçáo qualitativa pode ser executada por dols processas : O delta teste, baseado na distribuição de Gauss, e o $\mathrm{X}^{2}$-teste, baseado numa forma derivada das distribuiçąes de Pearson (BRIEGER, 1945).

I. No primeiro caso formamos um desvio relativo, divid"ndo a diferença entre a frequência observada e a frequencia esperada pelo seu erro standard. Uma vez que este altimo é calculado por uma formula geral, e não derivado dos valores observados, podemos atribuir-lhe um número infinito como gráu de liberdade. A formula é a seguinte :

delts (desvio relativo)

em números $=\frac{f(o b s)-p . N}{\sqrt{p(1-p) N}} \quad \frac{n f(1)=1}{n f(2)=\text { inf. }} . \quad .1 \mathrm{a}$

em percentagem $=\frac{\mathrm{f} \text { (obs.\%)-p\%}}{\sqrt{\frac{\mathrm{p} \%(100-\mathrm{p} \%)}{\mathrm{N}}}} \frac{\mathrm{nf}(1)=1}{\mathrm{nf}(2)=\mathrm{inf} .} \cdot \ldots 1 \mathrm{~b}$

Este teste pode evidentemente ser aplicado apenas na variação alternativa, onde $\mathbf{p}$ representa a frequencia ideal do tipo ou da classe esperada, enquanto que (1-p) representa a frequência do seu năo-aparecimento.

$\mathrm{Na}$ análise da variaçåo qualitativa múltipla, com numerasos tipos ou classes, temos que calcular um erro standard c um desvio relativo para cada classe.

Além disso o teste apenas pode ser aplicado quando $\mathrm{p} e$ um valor entre 0,1 e 0,9. Quando a frequéncia esperada for menor do que 0,1 a distribuição do binômio torna-se táo assimétrica que podemas substituí-la pela série de Poisson. Também neste caso podíamos calcular o desvio relativo, lembrando que agora a frequência esperada é representada pela média da 
série de Poisson m e que o erro standard de uma série de Poisson é igual a raí quadrada da média.

$$
\text { desvio relativo }=\frac{f(\mathrm{obs})-\mathrm{m}}{\sqrt{\overline{\mathrm{m}}}} \cdot \cdot 1(\mathrm{c})
$$

Porém este têrmo não tem utilidade na análise estatística, porque os seus limites de acuso são diferentes para cada série de Poisson, sendo pràticamente impossível dar táboas completas para todas as séries de Poisson.

II - O X2-teste pode ser aplicado em qualquer caso da variaçáo qualitativa tanto alternativa ou múltipla, calculando-se para cada classe o quociente do quadrado da difererça entre a frequência obseryada e esperada, dividido pela trequencia esperada :

em numeros :

$$
\mathbf{X}^{2}=\frac{\text { f(obs. })-\mathbf{f}(\text { esp. })^{2}}{f(\text { esp. })} \cdot .2 a
$$

em percentagem:

$$
X^{2}=\frac{\{f(\text { obs } \%)-f(\text { esp. } \%)\}^{2} N}{f(\text { esp. } \%) 100} \cdot .2 b
$$

Designando as probabilidades das diversas classes com as letras p1, p2, etc., devemos distinguir dois casos neste X2-teste.

II - a) : Se o valor de p1 referente a uma das classes, for muito grande, isto é, năo muito diferente de um, podemas desprezar o respectivo valor de $\mathrm{X}^{2}$, por ser em geral muito pequeno. Analisamos individualmente os valores de $\mathbf{X}^{2}$ de cada uma das classes restantes, cada uma com 1 grau de liberdade. podendo também somar as valores de $\mathrm{X}^{2}$ individuais. Como limite da aproximaçăo a um, podemos aceitar um valor de $p$ maior do que 0,9 , e como valor suficientemente pequeno para cada uma das demais classes podemos aceitar valores menores do que 0,1 .

II b) : Se de outro lado os valores de $p$ săo maiores do que 0,1 e menores do que 0,9 , náo será mais justificada uma análise simples dos quocientes $X^{2}$ individuais, mas será necessário estudar o $\mathrm{X}^{2}$ total, isto é, a soma de todos os valores individuais $\mathrm{X}^{2}$ total, isto é, a soma de todos os valores individuais $\mathrm{X}^{2}$, com grau de liberdade igual a seu número menos um. 
Devemas ainda lembrar uma limitação do X2-teste; ele pode ser aplicado sòmente quando a frequéncia esperada em números for igual a cinco pelo menos, ou maior.

Os dois testes, delta-teste e $\mathbf{X}^{2}$-teste, não representam testes diferentes. $O$ valor de $X-2$-teste para a variaçáo alternativa e para valores de $\mathrm{p}$ entre 0,1 e 0,9 , é igual ao quadrado de delta, calculado para os mesmos dados. Esta relação algébrica é fácil de demonstrar.

Supomos que temos duas classes com a e b individuas, sendo o total $\mathrm{N}$ igual a sua soma, e que as frequencias ideais såo iguais a pa e pb de modo que ( $\mathrm{pa}+\mathrm{pb}$ ) é igual a um, e beremos :

$$
\mathrm{X}^{2}=\frac{\left(\mathrm{a}-\mathrm{N} \mathrm{p}_{\mathrm{a}}\right)^{2}}{N \mathrm{p}_{\mathrm{a}}}+\frac{\left(\mathrm{b}-\mathrm{N} \mathrm{p}_{\mathrm{b}}\right)^{2}}{N \mathrm{p}_{\mathrm{b}}} \cdot \ldots 3
$$

Evidentemente os dois desvios têm um valor igual exceto o sinal que é oposto, como se vê pela deduçăo seguinte :

$$
\begin{array}{clr}
a+b=N & \text { ou } & a=N-b \\
p_{a}+p_{b}=1 & \text { ou } \quad p_{a}=1-p_{b} \\
\hline \text { temos: } a-N p_{a} & =(N-b)-N\left(1-p_{b}\right) \\
& =N p_{b}-b \\
& =-\left(b-N p_{b}\right)
\end{array}
$$

Agora podemos simplificar a equaçáo (3):

$$
\begin{aligned}
\mathrm{X}^{2} & =\left(\mathrm{a}-\mathrm{N} \mathrm{p}_{\mathrm{a}}\right)^{2} \cdot\left(\frac{1}{N \mathrm{p}_{a}}+\frac{1}{\mathrm{~N}\left(1-\mathrm{p}_{\mathrm{a}}\right)}\right) \\
& =\left(\mathrm{a}-\mathrm{N} \mathrm{p}_{\mathrm{a}}\right)^{2} \cdot \frac{\left(1-\mathrm{p}_{\mathrm{a}}\right)+\mathrm{p}_{\mathrm{a}}}{\mathrm{p}_{\mathrm{a}} \cdot\left(1-\mathrm{p}_{\mathrm{a}}\right) \mathrm{N}} \\
& =\frac{\left(\mathrm{a}-\mathrm{N} \mathrm{p}_{\mathrm{a}}\right)^{2}}{\mathrm{p}_{\mathrm{a}}\left(1-\mathrm{p}_{\mathrm{a}}\right) \mathrm{N}}=(\text { detta })^{2} \cdot \cdot \cdot 4
\end{aligned}
$$

Assim é apenas uma simples questáo de conveniência se usamos na variaçáo alternativa o delta teste com as limites bllaterais da distribuiçăo de Gauss ou o $\mathrm{X}^{2}$-teste com os limItes unilaterais da distribuiçăo de Pearson com $n f 1=1$; nf2 $=$ infinito. Os limites dêstés dois testes são idênticos.

As distribuiçóes de $\mathrm{X}^{2}$ podem ser transformadas 
em distribuições de Pearson, dividindo o valor $X^{2}$ pelo grau de liberdade nf1, que é no nosso caso igual a um, e extraindo a raiz quadrada. A distribuiçáo de Pearson com $n 1=1 ; n 2=$ infinito é igual a metade da distribuição de Gauss de modo que as limites unilaterais superiores desta distribulçáo de Pearson são iguais a as limites bilaterais superiores da distribulçåo de Gauss.

Esta derivação dá ainda a explicação matemática da razão pela qual as valores individuais de $X^{2}$ na variaçáo alternativa e com valores de $p$ entre 0,1 e 0,9 năo têm interesse, e que devemos dar maior importancia ao $\mathrm{X}^{2}$ total.

Os valores de $\mathrm{X}^{2}$ individual são quocientes do mesmo termc, do quadrado do desvio, dividido ou por p.N ou por (1-p)N. Quando $p=(1-p)=0,5$ os dols valores de $X^{2}$ sáo identicos e quando $\mathrm{p}$ for diferente de (1-p) um das valores de $\mathbb{X}^{2}$ tem que ser menor do que o outro, tratando-se de uma simples consequéncia algébrica, e náo de qualquer resultado estatístico. $\mathrm{Na}$ variação múltipla, com três ou mais classes, a situaçáo é diferente e aqui tanto as valores das $\mathrm{X}^{2}$ individuais como o do $X^{2}$ total tem Interesse estatistico.

Pelo exposto ficou claro que as distribuiçoes do $\mathbf{X}^{2}$, das quais săo calculados os limites de precisăo na execuçăo do teste, enumerados nas táboas em uso geral (veja táboa III), săo contínuas, e temos que demonstrar quando estes limites pode!n ser aplicados, pois as frequencias na variaçăo qualitativa năo săo contínuas, mas ao contrário valores descontínuos. Pordemos sempre obter 1,2,3.. Individuos de um determinado tipo, mas náo meio individuo ou qualquer outra fraçá. Assim temos antes de mais nada resolver qual o tipo de distribuiçáo descontínua seguida pelas frequencias observadas e quando podemns aplicar uma aproximaçăo a uma distribuição contínua.

Limitaremos a discussăo aos casos da variaçăo alternativa, sendo fácll de derivar depols a soluçăo para os casos mais complexas da variaçăo múltipla.

A variação da frequencla $p$ pode ser calculada pela expansăo do binomio.

$$
(p+q)^{N} \text { onde } p+q=1 \ldots . .
$$

aste termo binominal se aproxima a dols extremas:

Quando $p$ é bem pequeno, de modo que $q$ fica aprox:madamente igual a 1, a série binominal se transforma numa série de Poisson, com frequencia média $\bar{m}$ igual a ( $p$. N) e erro standard igual à raiz quadrada desta média $\overline{\mathrm{m}}$.

Se de outro lado $\mathrm{N}$ torna-se muito grande, aproximando-se 
ao infinito, a série descontínua binominal se transforma na distribuição contínua de Gauss, devendo ainda o valor de $\mathbf{N}$ ser tanto maior quanto mais $p$ e $q$ forem desiguais.

Para melhor ilustrar estas duas aproximaçס̃es, utiliza:einos os dados contidos nos Quadros 1 a 3, nos quais enconrramas as frequências de distribuições de Poisson na segunda coIuna a esquerda e as valores de $\mathrm{X}^{2}$-total, baseados na distribuicão modificada de Gauss, na segunda coluna da direita.

Nas duas colunas centrais encontramos as frequéncias de duas distribuiçőes binominais com $p=1 / 20$ ou 0,05 e com $\mathrm{p}=1 / 10$ ou 0,10 . Foram escolhidas frequências esperadas iguais a 5 (Quadro 1), 10 (Quadro 2) e 16 (Quadro 3). As linhas horizontais indicam a posição dos limites de $5 \%, 1 \%$ e $0,1 \%$ de precisão, isto é, os pontos que cortam uma área em ambas as extremidades das distribuiçőes de tal modo que a soma das duas áreas excluidas em ambas as extremidades é igual a 5\%, 1\% e 0,1\% da área total da distribuiçåo (compare BRIEGER, 1945 : Fig. 4).

A posição dos limites do binômio $(1|20+19| 20)$ N iterceira roluna) se aproxima muito aos limites da séries de Foisson (segunda coluna à esquerda) e aquela das limites do binómio $(1 ! 10+9 \mid 10) N$ se aproxima dos limites de $X^{2}$-total, para valores de $\mathrm{N}=50,100$ e 150 .

Fica assim demonstrado que é correta a conclusão de BRIEGER (1946), MOLINA (1943), e outros que os limites entre as séries de Poisson e as séries binominais podem ser fixados aproximadamente num valor de $p$ igual a 0,1 e que a série binominal se torna razoàvelmente contínua parcu expsentes maiores do que 50 (BRIEGER, 1945 : Fig. 1)

As distribuiçర̋es de Poisson são por si mesmas assimétricas, mas para que possamos aplicar como aproximação unia distribuição modificada de Gauss devemos exigir uma sinetria da distribução além da continuidade.

O X2-total do Quadro 1 mostra uma forte assimetria devida à pressáo do limite absoluto zero, pois o valor menor possivel da frequência observada é evidentemente zero, não podendo haver número de individuos com sinal negativo. Assim apesar do que a variação da frequência observada possa subir até qualquer valor, além de $0,1 \%$ limites de probabilidade, - valor mais extremo na outra extremidade é o zero, jusinmente localizado além do limite de $5 \%$ de probabilidade, ᄅ dentro do limite de $1 \%$.

O X2-total do Quadro 2, que se refere a uma frequencia esperada de 10 já não acusa mais uma assimetria causada pela pressão do limite absoluto zero.

Para melhor ilustrar estas relaçőes calculel os valores de $\mathrm{X}^{2}$ 
Individual que constam nos Quadros 4 a 6, para os valores da frequência esperada igual a 5,10 e 15 .

Com $f(e s p)$ igual a 5, temas uma variaçáo ainda bem assimétrica, podendo atingir apenas os desvios negativos do $1 \%$ limite de precisăo, limite este porém mutas vezes satisfatório. Com $f$ (esp) igual a 10 atinglmos justamente, no lado de desvios negativos, o $0,1 \%$ limite de precisăo e com $f$ (esp igual a 15 estamos pràticamente livres do efelto da pressăo do limite absoluto zero e da assimetria por este causada.

Histes Quadros dáo assim a razáo pela qual alguns autores admitem o emprégo do $\mathrm{X}^{2}$-teste para valores minimos da frequéncia esperada de pelo menos 5 (BRIEGER) ou de pelo menos 10 (SNEDECOR e outros).

Em resumo podemos formular a seguinte conclusão:

O X2-teste, $\mathrm{C}$ do mesmo modo o delta-teste na análise da variaçáo qualitativa, se baseiam na hipótese que as frequencias observadas variam em volta do valor da frequencia esperada e de acôrdo com uma distribuição modificada de Gauss. Uma aproximaçăo à distribulçăo de Gauss, pode ser aplicada a priori apenas a) quando a frequência esperada em fração é maior do que 0,1 . b) quando a frequencia esperada em números é maior do que 5. c) quando o numero total que corresponde ao expoente do têrmo binominal, é maior do que 50 .

thstes tres valores criticos podemos reunir numa equação:

$$
\begin{aligned}
\mathrm{f}(\mathrm{obs}) & =\mathrm{N}-\mathrm{p} \\
\mathbf{5} & =50-0,1 \ldots \ldots \ldots \ldots \ldots \ldots \ldots 6
\end{aligned}
$$

Assim surgem dois problemas que discutiremos nos capitulas seguintes : a) como analisar amostras onde a frequencla esperada, em fraçóes, for menor do que 0,1.

b) como analisar amostras com $p$ maior do que 0,1 , mas onde o número total for menor do que 50, de modo que a frequencia esperada em números é menor do que 5 .

\section{II - Amostras com a frequência esperada $p$ menor do que 0,1}

A relaçăo entre 0 valor $X^{2}$ individual e a distribuiçăo de Polsson é fàcllmente demonstrada.

Lembrando-se que a frequência esperada numa série do Poisson é igual à média da sérle, $\mathrm{m}$, e que o erro standard é Igual à raiz quadrada desta média, podemos formular o desvio relativo : 
desvio relativo (Polsson) $=\frac{f(\text { obs. })-f(\text { esp })}{\text { erro }}=\frac{f(\text { obs })-\bar{m}}{\sqrt{\bar{m}}}$ (desvio relativo $\left.2=\frac{f(o b s)-\bar{m}^{2}}{m}=\frac{\{(\mathrm{f}(\text { obs })-f(e s p)}{\mathrm{m}}\right\}^{2}=\mathrm{X}^{2} \ldots 7$

Assim torna-se evidente que podemas aplicar o têrmo $\mathrm{X}^{2}$, mas devíamos empregar no teste de significancia limites d? precisão calculados de uma série de Poisson, em vez da distribuição modificada de Gauss.

A diferença entre as limites para a frequência observada calculada nas duas hipóteses consta no quadro 7.

Se nós compararmos a diferença entre o limite de $0,1 \%$ precisão da đistribuiçăo de Poisson e do $\mathrm{X}^{2}$-teste, para diferentes valores de $f(e s p)$ notamas que as diferenças em números variam pouco. Mas não devemos esquecer que uma divergência de dois individuos (última linha) representa uma inexatidăo de $4 \%$ apenas em relaçáo a frequencia esperada de 50 , quando uma aivergência de 3 individuas (na primeira linha) representa uma inexatidão de $300 \%$ em relação à frequencia esperada de 1.

Como inexatidáo ainda tolerável podemos aceitar os valores contidas na linha que corresponde a uma frequência esperada de pelo menos 5 ou melhor ainda de pelo menos 10 .

A explicação teórica da passibilidade de empregar na análise duma série de Poisson os limites da distribuiçáo de Gauss consiste no seguinte: um fato matemático bem conhecido que mesmo as distribuições de Poisson, como qualquer distribuição binominal assimétrica, tendem para a continuidade e simetria quando o expoente $\mathbf{N}$ torna-se bastante grande. No nasso caso podemos calcular fàcilmente éste valor $\mathbf{N}$ pela equaçăo

$$
\mathbf{f}(\text { obs. })=\text { N.p }
$$

e substituindo por $f(o b s)$ o valor 10 e para $p$ o valor 0,1 , $n$ qual nós aceitamos como limites entre as distribuiçz̃es de Poisson e do Binomio:

$$
\begin{aligned}
10 & =\mathrm{N} \cdot 0,1 \\
\mathrm{~N} & =100
\end{aligned}
$$

Se o valor de $p$ for menor ainda, podemos até aceitar o valor 5 como limite inferior para a frequencia esperada em núméros : com precisăo de $5 \%$ ou 0,05 : 


$$
\begin{aligned}
5 & =\mathrm{N} .0,05 \\
\mathrm{~N} & =100
\end{aligned}
$$

Os valores exatos dos limites de $f(o b s)$, calculados na base da distribuição de Polsson, estắo contidos na táboa 1. Incluimos nesta táboa os limites para as frequencias esperadas de $p$ igual a 0,001 até 15 .

\section{A aplicação da táboa é fàcilmente explicada :}

Esperamos por exemplo um acontecimento com uma frequéncia de $p=0,005$ e estudámos 60 individuos ao todo. Assim teremos uma frequencia esperada de 0,005 vezes 60 ou 0,3 in dividuas. Da sétima linha da táboa podemos deduzir que em 95 casos entre 100 obteremos qualquer valor entre 0 e 2 inclusive, isto é : 0 ou 1 ou 2 indivíduos do tipo esperado. Apenas 5 vezes em 100 repetiçðes (limite de $5 \%$ precisăo) podemos obțer 3 ou mais indivíduas, e uma vez em 1000 repetiçóes (limite de $0,1 \%$ de precisão) obteremas 4 individuos ou mais do tipo.

Invertendo a pergunta, e obtendo num experimento 4 ou mais individuos, podemos concluir que este desvio do valor esperado 0,3 é causado por agentes especials ou que a nossa hipótese básica era errada e que 0 valor esperado na realidade năo era 0,005 , mas que era outro e maior. Num outro trabalho recente (BRIEGER, 1947) já fol dada a soluçăo dêste último problema, isto é, como num caso déste achar o valor da frequêncla esperada mais provável.

A táboa $\tau$ vai apenas até valores da frequencia esperada igual a 15, pois como já fol explicado para valores maiores podemos sempre aplicar o $\mathrm{X}^{2}$-teste na sua forma comum.

\section{III - Amostras com frequência esperada maior do que 0,1 e número total menor do que 50.}

Já explicảmos acima na equação (4) que o $\mathrm{X}^{2}$-teste para duas classes é idêntico ao delta-teste, mas que năo é justificável a príori, substituir nos cálculos dos limites de precisăo uma distribuiçăo de Gauss para binômios com expoentes relativamente pequenos.

A dimensão das divergências entre os limites verdadeiros de distribuiçסes binominais e os limites de $\mathrm{X}^{2}$ calculados com aproximação de Gauss ficam ilustradas pelos dadas contidos nos Quadros 8 e 9. 
Os limites são pràticamente idênticos para o binômio $\left(\frac{1}{2}+\frac{1}{2}\right)^{N}$ exceto algumas pequenas divergências sem muita importáncia. Assim podemos aplicar o teste na forma comum e sem respeitar o limite de pelo menos 5 individuos para a frequência esperada. Apenas não devemos esquecer que em amostras menores, ficamos com uma variação assimétrica e pràticamente unilateral. Devido à pressăo do limite zero, náo podemos obter de forma alguma desvios negativos significantei quando o número esperado é menor do que 4.

A situação é sòmente pouco diferente para uma distribuição levemente assimétrica como o binômio $\left(\frac{1}{4}+\frac{3}{4}\right)^{N}$ (Quadro 9).

Mesmo quando descemos até frequenclas esperadas iguais a 1,5 ou 2 ou a números totais de individuas iguais a 6 ou 8 , encontramos apenas uma diferença de no máximo um individuo nos limites, calculados pelo dois processos.

Apesar de que podemos assim práticamente aplicar o $\mathbf{X}^{2-}$ teste na variação alternativa com duas classes, mesmo para as menores amostras que podem acontecer em experimentos, cal . culei para êstes dois binômios os limites exatos de precisz̃o e que constam na táboa II. A sua aplicaçăo năo precisa de nenhuma explicação detalhada.

\section{IV - Testes da homogeneidade de distribuições internas}

Além dos casos da variação qualitativa, o $\mathrm{X}^{2}$-teste é amplamente aplicado na análise estatistica quando queremos comparar as frequências em distribuiçōes observadas com aquelas em distribuiçoses teóricas adequadas, num teste que chamamas de teste da distribuição inteira.

Como exemplo escolhi a análise das médias do diametro de frutas em 217 árvores de laranja Baianinha, obtidas pelo enxêrto de borbulhas da mesma árvore matriz em numerosos cavalos de laranja azéda (BRIEGER, MOREIRA, 1914). Pociemos esperar que a variaçáo déstes diametros acompanhasse a distribuição de Gauss, e damos no Quadro 10 as frequências para intervalos tanto em fraçס̃es do êrro standard como em milímetros.

$\mathrm{Na}$ terceira coluna constam as frequências esperadas de acôrdo com a distribuiçăo de Gauss enquanto na quarta constam as frequencias observadas exporimentalmente.

Aplicando agora o $\mathrm{X}^{2}$-teste encontramos uma situaçăo classificada na introduçáo como o caso IIb. Para as oito clas- 
ses centrais com frequências esperadas maiores pod mos calcular o $\mathrm{X}^{2}$ sem hesitação. Para as demais classes devemos antes verificar se os valores de frequencia esperada são maiores do que 5. Verificamos que este náo é o caso para cinco classes em cada extremidade, de modo que temos que acumula: as suas frequências das extremidades para dentro até atingir - número mínimo necessário. Sómando as cinco classes chegámos a um valor da frequericia esperada igual a 4,92 ou quase igual a 5. Agora podemos executar o $\mathrm{X}^{2}$-teste. Comparando o total dos 10 valores de $\mathrm{X}^{2}$, com 9 graus de liberdade, com os limites que constam na táboa III, verificamos que êle é altamente significante e comparando os valores de $\mathrm{X}^{2}$ individuais, com um grau de liberdade cada um, constatamos que apenas as valores do $\mathrm{X}^{2}$ referentes às classes extremas não significantes. Assim sabemos que a anormalidade da distribulção observada consiste na alta frequencia de valores nas classes com desvios mais extremos, sejam das maiores do que $+2,00$ vezes o erro sandard, ou menores do que $-2,00$ vezes o erro standard.

O emprêgo da táboa I nas permite ir além déste resultado do $\mathrm{X}^{2}$-teste. $\mathrm{Na}$ terceira classe, correspondendo ao intervalo de $-3,5$ até $-4,5$ esperamos 0,04 valores e temos nenhum, na classe seguinte esperamos 0,24 e temos um, sendo amba:; as frequências observadas dentro dos limites de precisåo dado na Táboa I.

$\mathrm{Na}$ classe seguinte de $-2,5$ até $-3,0$ esp zramos 1,03 valores e os limites que nas indicam quais as frequencias que na devem ser mais encontradas săo, de acőrdo com a Táboa I.

$$
\begin{array}{r}
5 \% \text { precisáo : } 4 \\
1 \% \text { precisăo : } 5 \\
0,1 \% \text { precisǻo : } 7
\end{array}
$$

Assim o valor da frequênc'a observada 7 é fora do $0,1 \%$ de precisáo e portanto altamente significante.

Pelo mesmo processo podemos também provar que a an n. $^{-}$ malia da outra extremidade de distribuiçăo é devido às frequênclas no intervalo de $+2,5$ até $+3,0$ vezes o erro standard.

Ass:m empregando, os limites contidos na nova Táboa I, depois da aplicaçăo do $\mathrm{X}^{2}$-teste que é um teste muito sumário para as extremidades da distribuiçáo, podemos obter resultados mais exatos e detalhados. 


\section{RESUMO}

Na aplicação do $X^{2}$-teste devemos distinguir dois casas :

A) Quando as classes de variáveis sâo caracterizadas por frequências esperadas entre $\mathrm{p}=0,1 \mathrm{e} \mathrm{p}=0,9$, podemos apiicar o X2-teste pràticamente sem restriçăo. talvez aconselhável, mas náo absolutamente necessário limitar o teste aas casos nas quais a frequência esperada é pelo menos igual a 5. e porisso incluimos na Táboa II os limites da variaçăo de dois binomios $\left(\frac{1}{2}+\frac{1}{2}\right)^{\mathrm{N}}$ e $\left(\frac{1}{4}+\frac{3}{4}\right)^{\mathrm{N}}$ para valores pequenos de $\mathrm{N}$ e nos três limites convencionais de precisão : $5 \%, 1 \%$ e $0,1 \%$.

Neste caso, as valores dos $\mathbf{X}^{2}$-individuais tem apenas valor limitado e devemos sempre tomar em consideração principaimente o $\mathrm{X}^{2}$ total.

O valor para cada $\mathbf{X}^{2}$ individual pode ser calculado po" qualquer das expressరoe seguintes:

$$
\begin{aligned}
x^{2} & =\frac{(f \text { obs }-f \text { esp })^{2}}{f \text { esp. }} \\
& =\frac{(f \text { obs }-p N)^{2}}{p N} \\
& =\frac{(f \text { cbs } \%-p \%) .2 N}{p \% .100}
\end{aligned}
$$

O delta-teste dá o mesmo resultado estatístico como o $\mathbf{X}^{2}$ teste com duas classes, sendo o valor do X2-total algébricamente igual as quadrado do valor de delta. Assim pode ser mais fácil às vezes calcular o $\mathrm{X}^{2}$ total como quadrado do desvio relativo da variação alternativa :

$$
\begin{aligned}
x^{2} & =\frac{(f \text { obs }-p N)^{2}}{p .(1-p) \cdot N} \\
& =\frac{(f \text { obs } \%-p \%)^{2} . N}{p \% \cdot(100-p \%)}
\end{aligned}
$$


B) Quando há classes com frequência esperada menor do que $\mathrm{p}=0,1$, podemos analisar os seus valores individuais de $\mathrm{X}^{2}$, e desprezar o valor $\mathrm{X}^{2}$ para as classes com $\mathrm{p}$ maior do que 0,9. $O X^{2}$-teste, todavia, pode agora ser aplicado apenas, quando a frequencia esperada for pelo menas igual ou maior do que 5 ou melhor ainda, igual ou maior do que 10.

Quando a frequencla esperada for menor do que 5, a variaçáo das frequencias observadas segue uma distribuiçăo de Poisson, não sendo possivel a sua substituiçăo pela aproximação Gausseana.

A táboa I dá as limites da variaçáo da série de Poisson para frequencias esperadas (em números) desde 0,001 até 15 .

A vantagem do emprégo da nova táboa I para a comparaçáo, classe por classe, entre distribuiçб̋es esperadas e observadas é explicada num exemplo concreto. Por melo desta táboa obtemos informaçбes muito mais detablhadas do que pelo $\mathrm{X}^{2-}$ teste devido ao fato que neste último temos que reunir as classes nas extremidades das distribuições até que a frequência esperada atinja pelo menos o valor 5 .

Incluimas como complemento uma táboa dos limites $\mathrm{X}^{2}$, para 1 até 30 graus de liberdade, tirada de um outro trabalho recente (BRIEGER, 1946). Para valores maiores de graus de liberdade, podemos calcular os limites por dois processos:

Podemas usar uma solução dada por Fischer:

$$
\sqrt{2 \mathrm{X}^{2}}-\sqrt{2 \mathrm{nf}}=\text { delta }
$$

Devem ser aplicados os limites unilaterais da distribuiçãc de Gauss : $5 \%: 1,64 ; 1 \%: 2,32 ; 0,1 \%: 3,09$ :

Uma outra solução podemos obter segundo BRIEGER (1946) calculando o valor :

$$
\sqrt{\frac{\mathrm{X}^{2}}{\mathrm{nf}}}=\text { teta }
$$

e procurando os limites nas táboas para limites unilaterais de distribuiçס̃es de Flscher, com $n 1=n f\left(X^{2}\right) ; n 2=$ inf; (BRIEGER, 1946). 


\begin{abstract}
The main object of the paper is a revision of the methods for the statistical analysis of qualitative variation in small samples. In general, this analysis is carried out by means of two tests, which are mathematically different, but give identical statistical results: the analysis of relative deviates, i.e. of te quotients between the deviate of the observed frequancy, with regerds to the expected frequency divided by its stalidard error, or an analysis using the $\mathrm{X}^{2}$-test.

We must distinguish, in our discussion, two cases, which are quite different. The basic distribuition of all cases of qualitative variation is the binomial. If we expect any qualitative result to occur with probability $p$,its non-occurence having probability q equal to (1-p), the expectancy to have, $0,1,2 \ldots \mathrm{m}$ cases or individuals of the expected type in $\mathrm{N}$ trials may be calculated by expanding the binomial.
\end{abstract}

$$
(p+q)^{N}
$$

Such a binomial may be substituited by other distribuitions in two special cases:

a) If $p$ is very small and thus $q$ is aproaching the value one, we may substitute the binomial by a Poisson series.

b) If the exponent $\mathbf{N}$ becomes very large, the binomial is approaching a continous distribution, i.e. the normal or Gaussean distribution.

Only in the second case, the application of the $\mathrm{X}^{2}$-test is really justified, and in all other cases, we are dealing only with approximations. Indvidual values of $\mathrm{X}^{2}$ - should follow a mo.. dified distribution of Pearson, with $n 1=1 ; \mathrm{n} 2=$ inf. Since this distributon corresponds exactly to one half of the Gaussean dstribution, it follows that the b'lateral limits of the latter are equal to the unilateral limits of the former. These points have been explained fully elsewhere (BRIEGER, 1945, 1946).

We have now to decide which value of $p$ may be accepted as a satisfactory limit between a Poisson and a binomial series. Quadros 1-3, show that the conventional limit of $p=0,1$ is fully justified, from a practical point of view. In these tables we find in the second column from the left the frequencies of a Poisson series, and in the second column from the night the values of $X^{2}$ based, as cxplained, on a modified Gaussean 
distribution. The two columns in the centre correspond to two binomials and it is evident that the first with $p=0,05$ has its limits of precision almost at the same level as the Poisson series, while the other with $p=0,1$ agrees fairly well with the limits of the $X^{2}$ series. Thus is seems justified to treat separately the caises with expected frequencies of $p$ equal or smaller that 0,1 and those with $p$ larger than 0,1 .

A) When the different classes, wich may be two (alternative variability) or more (multiple variability, have all expected frequences of $p$ between 0,1 and 0,9 , we may use practically the $X^{2}$ test with out any restriction. Quadros 8 and 9 show that the limits calculated for two binomials are practically identical with those of the $X^{2}$ total.

Nevertheless a special table is given (table 11) for the limits of binomials with $p$ equal 0,5 and 0,25 and expected class frequencies of less than 10.

One must not forget that in these cases the individual values of $\mathrm{X}^{2}$ for each class are of less importance than their sum, the $\mathrm{X}^{2}$ total.

The value of $X^{2}$ for each class may be calculated either with the general formula, using actual numbers or with a modified formula using percentages :

$$
\begin{aligned}
X^{2} & =\frac{(f \text { obs }-f \text { esp })^{2}}{f \text { esp }}=\frac{(f \text { esp }-N p)^{2}}{N p} \\
& =\frac{(f \text { obs } \%-p \%)^{2} \cdot N}{p \% \cdot 100}
\end{aligned}
$$

In the case of alternative variabllity, we may calculate directly the value of the $X^{2}$ total, by squaring the relative aeviate :

$$
\begin{aligned}
X^{2} \text { total } & =\frac{(f \text { obs }-f \text { esp })^{2}}{p \cdot(1-p) \cdot N}=\frac{(f \text { obs }-N p)^{2}}{p \cdot(1-p) \cdot N} \\
& =\frac{(f \text { obs } \%-p \%)^{2} \cdot N}{p \% \cdot(100-p \%)}
\end{aligned}
$$


B) If we have one or more classes with expected freque11cies equal os smaller than 0,1 we have to deal with a Poissor: series. As shown in Quadro 7 the agreement between the limits of the Poisson series and the $\mathbf{X}^{2}$-test for one classe (simple $\mathrm{X}^{2}$ ) is only satisfactory when the expected frequency. is larger than 10 and tolerable when it is between 5 and 10. If the expected numbe: should be smaller still, we cannot use anymore the $X^{2}$-test, but should use the values given in table $I$, calculated for Poisson series witr expected frequencies (in numbers) from 1 to 15 .

Very frequently the $\mathrm{X}^{2}$-test is used for comparing in detail observed and expected distributions, a test called sometimes "homogeneity test". Since generally the frequencles in the marginal classes are less than five, we have to accumulate by summing the frequencies from the more extreme clisses towards the center, untill all accumulated and remaining values are at least equal to five. The statistical information. lost in this accumulating process, may be recovered when comparing the individual class frequencies with the limiting values in table 1. As ilustration, a concrete exemple is discussed. (Quadro 10).

The formulas and tables of this paper have been tried out first during sometime and, having been found of considerable value in the execution of statistical analysis, are now published. In order to permit a more general use, a table of ordinary limits for the $X^{2}$-test is included, taken from a recent paper (BRIEGER, 1946).

\section{LITERATURA}

BRIEGER, F. G. - 1945 - As distribuiçōes do Acaso - Anais da Escola, Vol: II, pp. 321-391.

BRIEGER, F. G. - 1946 - Limites Unilaterais e Bilaterais na amostra estatística - Bragantia - Vol : VI, pp. 479-545.

BRIEGER, F. G. - 1947 - A determinaçáo das números de individuos mínimos necessários na Experimentação Genética - Anair da Escola. Vol : IV (em impressão).

MOLINA, E. C. - 1943 - Poisson's Exponential Binominal LImits - Van Nostrand Co. - New York. 


\section{QUADRO 1}

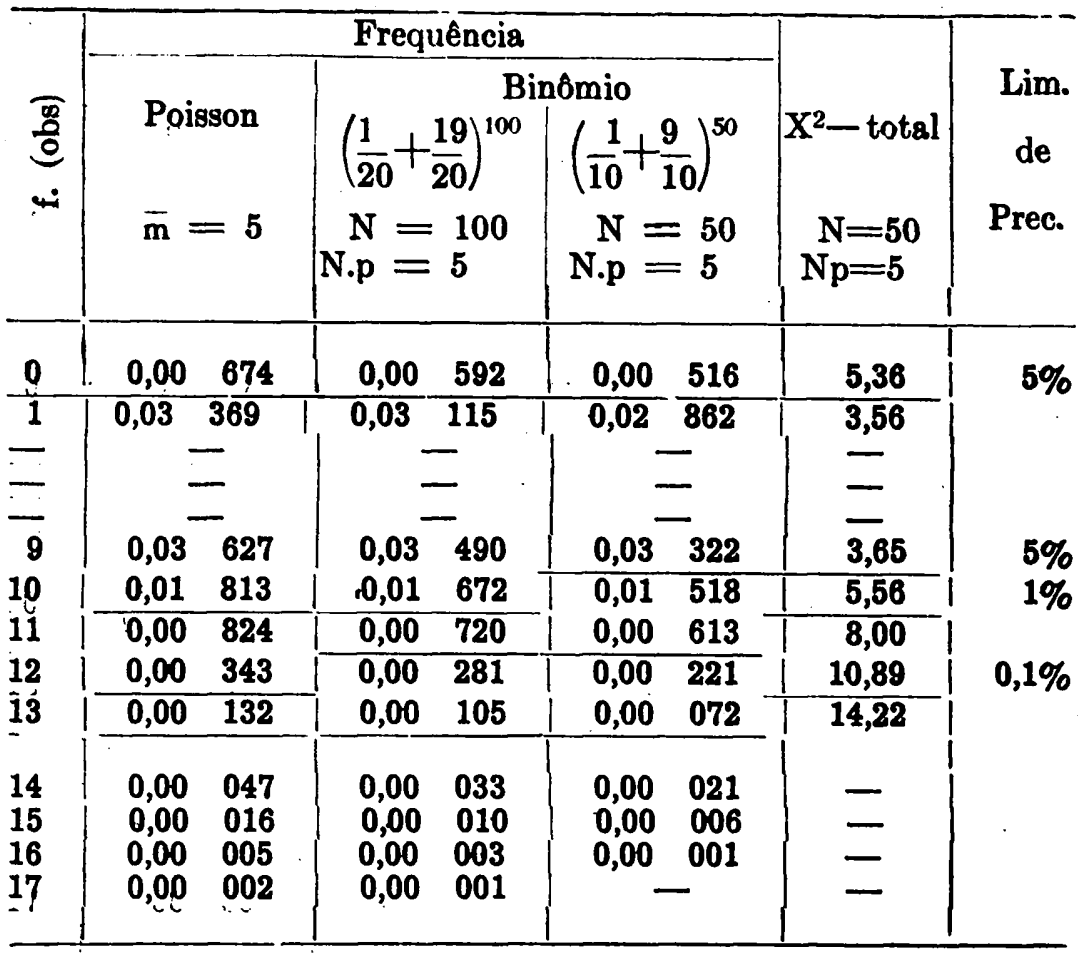




\section{QUADRO 2}

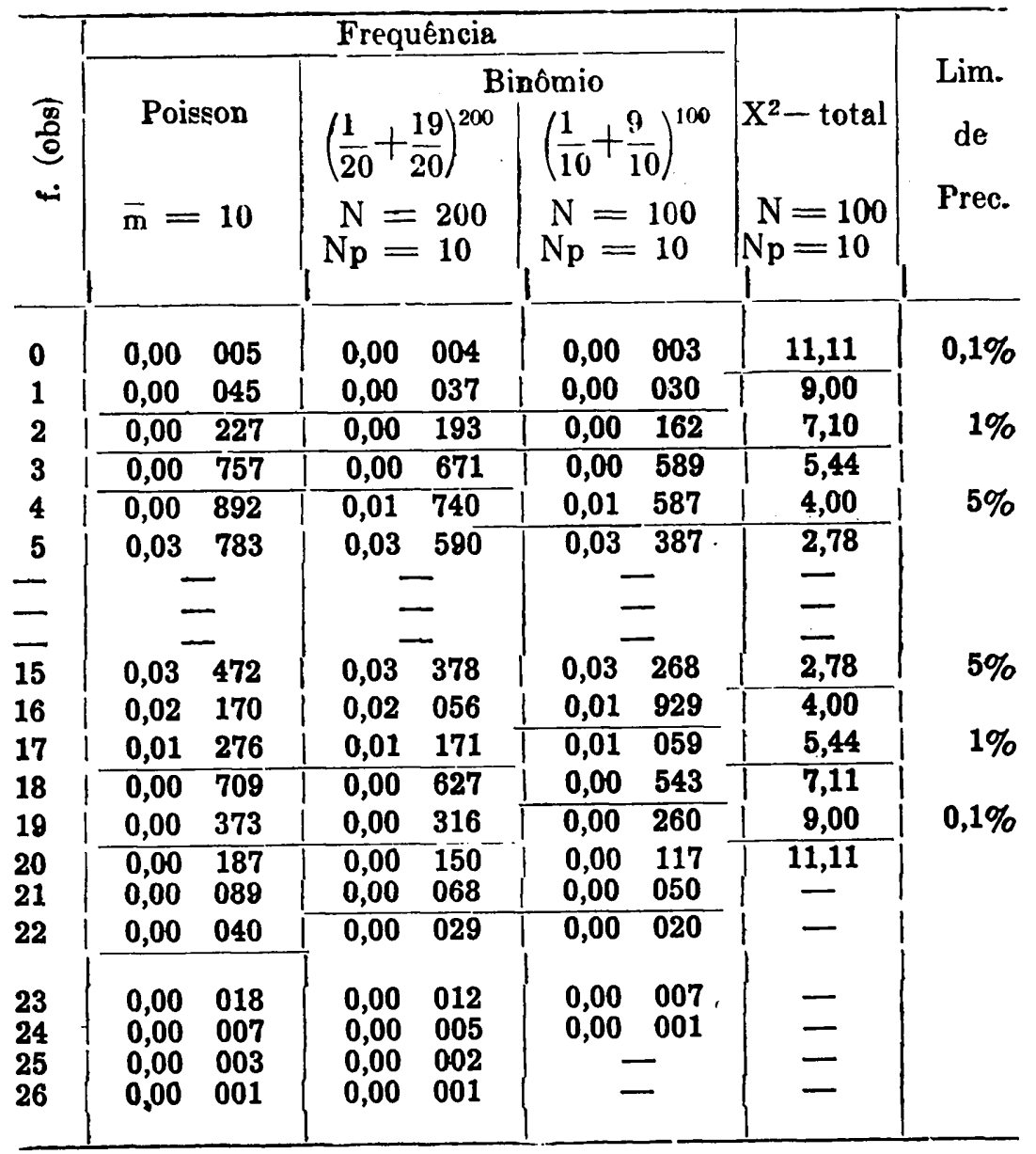




\section{QUADRO 3}

\begin{tabular}{|c|c|c|c|c|c|c|}
\hline \multirow{4}{*}{ ن } & \multicolumn{4}{|c|}{ Frequência } & \multirow{4}{*}{$\begin{array}{c}\mathrm{X}^{2}-\text { total } \\
\mathrm{N}=150 \\
\mathrm{~Np}=15\end{array}$} & \multirow{4}{*}{$\begin{array}{c}\text { Lim. } \\
\text { de } \\
\text { Prec. }\end{array}$} \\
\hline & \multicolumn{2}{|c|}{ Poisson } & \multicolumn{2}{|c|}{ Binomio } & & \\
\hline & \multirow{2}{*}{\multicolumn{2}{|c|}{$\overline{\mathrm{m}}=15$}} & \multirow{2}{*}{$\begin{array}{l}\left(\frac{1}{20}+\frac{1 y}{20}\right)^{200} \\
N=300 \\
N p=15\end{array}$} & \multirow{2}{*}{$\begin{array}{l}\left(\frac{1}{10}+\frac{9}{10}\right)^{150} \\
N=150 \\
N p=15\end{array}$} & & \\
\hline & & & & & & \\
\hline 0 & \multicolumn{2}{|c|}{ 二 } & - & 二 & - & \multirow{3}{*}{$0,1 \%$} \\
\hline 2 & 0,00 & 003 & 0,00 & 0,00 & $1 \overline{12.52}$ & \\
\hline 3 & 0,00 & 017 & $0,00 \quad 013$ & 0,00 & 10,67 & \\
\hline $\begin{array}{l}4 \\
5\end{array}$ & $\begin{array}{l}0,00 \\
0,00\end{array}$ & $\begin{array}{l}054 \\
194\end{array}$ & $\begin{array}{ll}0,00 & 053 \\
0,00 & 164\end{array}$ & $\begin{array}{ll}0,00 & 041 \\
0,00 & 137\end{array}$ & $\begin{array}{l}8,96 \\
7,34\end{array}$ & \multirow{2}{*}{$1 \%$} \\
\hline $\begin{array}{l}6 \\
7\end{array}$ & $\begin{array}{l}0,00 \\
0,01\end{array}$ & $\begin{array}{l}484 \\
037\end{array}$ & $\begin{array}{ll}0,00 & 425 \\
0,00 & 939\end{array}$ & $\begin{array}{ll}0,00 & 368 \\
0,00 & 842\end{array}$ & $\begin{array}{l}5,33 \\
4,74\end{array}$ & \\
\hline 8 & 0,01 & $9 \overline{44}$ & 0,01810 & $0,01 \quad 672$ & 3,64 & \multirow{5}{*}{$5 \%$} \\
\hline - & & - & $=$ & - & \\
\hline 一 & \multicolumn{2}{|c|}{ - } & 一 & 二 & Z & \\
\hline 22 & 0,02 & 036 & $0,01 \quad 940$ & 0,01 & 3,64 & \\
\hline 23 & 0,01 & 328 & $0,01 \quad 234$ & $0,01 \quad 133$ & 4,74 & \\
\hline 24 & 0,00 & 830 & $0,00 \quad 750$ & $\overline{0,00} \quad 666$ & 5,33 & $1 \%$ \\
\hline 25 & 0,00 & 498 & $0,00 \quad 436$ & $0,00 \quad 373$ & 7,34 & \\
\hline 26 & 0,00 & 287 & $0,00 \quad 242$ & 0,00 & 8,96 & \\
\hline 27 & 0,00 & 160 & 0,00 & 0,00 & 10,67 & $0,1 \%$ \\
\hline 28 & 0,00 & 086 & 0,00 & 0,00 & 12,52 & \\
\hline 29 & 0,00 & 044 & $0,00 \quad 033$ & $0,00 \quad 023$ & - & \\
\hline 30 & & & & & - & \\
\hline 31 & 0,00 & 011 & $0,00 \quad 007$ & $0,00 \quad 004$ & - & \\
\hline 32 & 0,00 & & $0,00 \quad 003$ & $0,00 \quad 002$ & 一 & \\
\hline 33 & 0,00 & 002 & $0,00 \quad 001$ & $0,00 \quad 001$ & - & \\
\hline 34 & 0,00 & 001 & & & - & \\
\hline
\end{tabular}


QUADRO 4

Limites do $\mathrm{X}^{2}$ individual (uma classe)

\begin{tabular}{|c|c|c|c|c|}
\hline \multicolumn{5}{|c|}{ f.esp) 5} \\
\hline \\
\hline
\end{tabular}


QUADRO 5

Limites do $\mathrm{X}^{2}$ individual (uma classe)

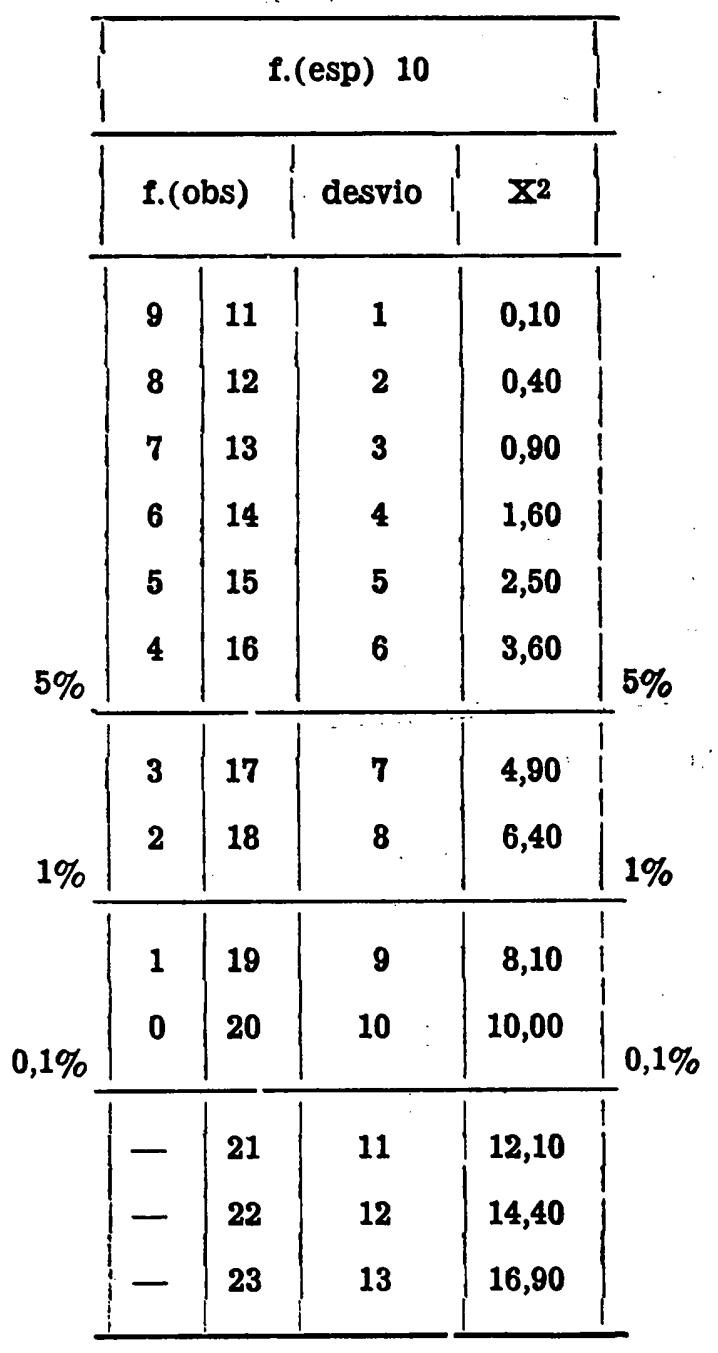


QUADRO 6

Limites do $\mathrm{X}^{2}$ individual (uma classe)

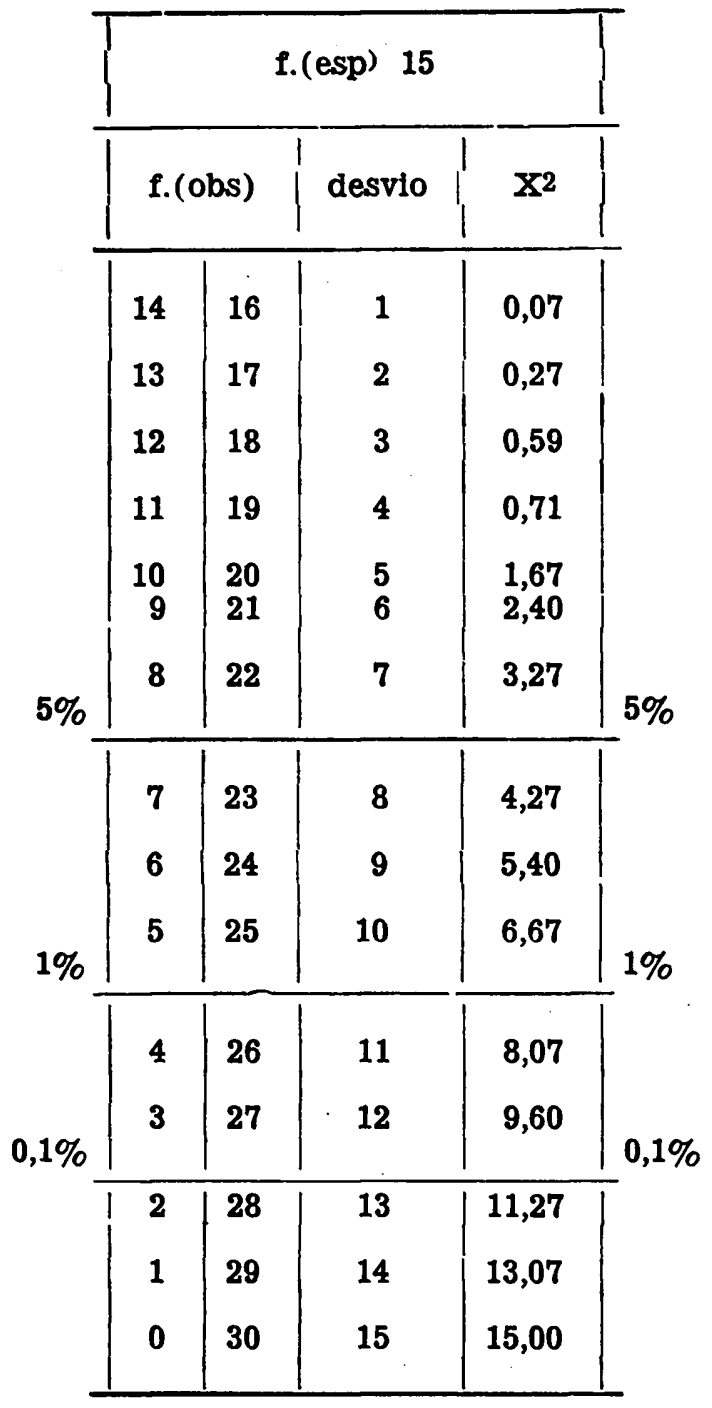


QUADRO 7

Comparação dos limites de $\mathbf{f ( o b s )}$ para a distribuição de Poisson e $0 \mathrm{X}^{2}$-teste com uma classe

\begin{tabular}{|c|c|c|c|c|c|c|c|}
\hline \multirow{3}{*}{ f.esp } & \multirow{3}{*}{ Fórmula } & \multicolumn{2}{|c|}{ Precisão 5\% } & \multicolumn{2}{|c|}{ Precisão $1 \%$} & \multicolumn{2}{|c|}{ Prec. $0,1 \%$} \\
\hline & & \multicolumn{6}{|c|}{ Limites de $f(e s p)$} \\
\hline & & Inf. & Sup. & Inf. & Sup. & Inf. & Sup \\
\hline \multirow[t]{2}{*}{1,0} & Polsson & - & 4 & - & 5 & - & 7 \\
\hline & $\mathrm{X}^{2}$ simples & - & 3 & - & 4 & - & 4 \\
\hline \multirow[t]{2}{*}{1,5} & Poisson & - & 5 & - & 6 & - & 8 \\
\hline & $\mathrm{X}^{2}$ simples & - & 4 & - & 5 & - & 6 \\
\hline \multirow[t]{2}{*}{2,0} & Poisson & 一 & 6 & - & 7 & - & $\mathrm{g}$ \\
\hline & $\mathrm{X}^{2}$ simples & - & 5 & - & 6 & - & 7 \\
\hline \multirow[t]{2}{*}{2,5} & Poisson & - & 7 & - & 8 & - & 10 \\
\hline & $\mathrm{X}^{2}$ simples & - & 6 & - & 7 & - & 8 \\
\hline \multirow[t]{2}{*}{3,0} & Poisson & - & 8 & - & 9 & - & 11 \\
\hline & $\mathrm{X}^{2}$ simples & - & 7 & - & 8 & - & 8 \\
\hline \multirow[t]{2}{*}{$\mathbf{3 , 5}$} & Poisson & - & 9 & - & 10 & - & 12 \\
\hline & $\mathrm{X}^{2}$ simples & - & 8 & - & 9 & - & 10 \\
\hline \multirow[t]{2}{*}{4,0} & Poisson & $\mathbf{0}$ & 9 & - & 11 & - & 13 \\
\hline & $\mathrm{X}^{2}$ simples & $\mathbf{0}$ & 8 & - & 10 & - & 11 \\
\hline \multirow[t]{2}{*}{$\mathbf{4 , 5}$} & Poisson & 0 & 10 & - & 12 & - & 14 \\
\hline & $\mathrm{X}^{2}$ simples & $\mathbf{0}$ & 9 & - & 10 & - & 12 \\
\hline \multirow[t]{2}{*}{$\mathbf{5}$} & Poisson & 0 & 11 & - & 13 & - & 15 \\
\hline & $\mathrm{X}^{2}$ simples & 0 & 10 & - & 11 & - & 13 \\
\hline \multirow[t]{2}{*}{6} & Polsson & 1 & 12 & 0 & 14 & 一 & 17 \\
\hline & $\mathrm{X}^{2}$ simples & 1 & 11 & - & 13 & - & 15 \\
\hline \multirow[t]{2}{*}{7} & Poisson & 1 & 14 & 0 & 16 & - & 18 \\
\hline & $\mathrm{X}^{2}$ simples & 1 & 13 & 0 & 14 & - & 16 \\
\hline \multirow[t]{2}{*}{8} & Poisson & 2 & 15 & 1 & 17 & 0 & 20 \\
\hline & $\mathrm{X}^{2}$ simples & 2 & 14 & 0 & 16 & - & 18 \\
\hline \multirow[t]{2}{*}{9} & Poisson & 3 & 16 & 1 & 19 & 0 & 21 \\
\hline & $\mathrm{X}^{2}$ simples & 3 & 15 & 1 & 17 & - & 19 \\
\hline \multirow[t]{2}{*}{10} & Poisson & 3 & 18 & 2 & 20 & 1 & 23 \\
\hline & $\mathrm{X}^{2}$ simples & 3 & 17 & 1 & 19 & - & 21 \\
\hline \multirow[t]{2}{*}{11} & Poisson & 4 & 19 & 3 & 21 & 1 & 24 \\
\hline & $\mathrm{X}^{2}$ simples & 4 & 18 & 2 & 20 & 0 & 21 \\
\hline \multirow[t]{2}{*}{12} & Poisson & 5 & 20 & 3 & 23 & 1 & 26 \\
\hline & $\mathrm{X}^{2}$ simples & 5 & 19 & 2 & 22 & 0 & 24 \\
\hline \multirow[t]{2}{*}{15} & Poisson & 7 & 24 & 5 & 27 & $\mathbf{3}$ & 30 \\
\hline & $\mathrm{X}^{2}$ simples & 7 & 23 & 5 & 25 & 2 & 28 \\
\hline 20 & Poisson & 11 & 30 & 9 & 33 & 6 & 37 \\
\hline & $\mathrm{X}^{2}$ simples & 11 & 29 & 8 & 32 & 5 & 35 \\
\hline 30 & Poisson & 19 & 42 & 16 & 46 & 13 & 51 \\
\hline & $\mathrm{X}^{2}$ simples & 19 & 41 & 15 & 45 & 12 & 48 \\
\hline 40 & Poisson & 27 & 54 & 24 & 58 & 20 & 63 \\
\hline & $\mathrm{X}^{2}$ simples & 27 & 53 & 23 & 57 & 19 & 61 \\
\hline 50 & $\begin{array}{l}\text { Poisson } \\
\mathrm{X}^{2} \text { simples }\end{array}$ & $\begin{array}{l}36 \\
36\end{array}$ & $\begin{array}{l}66 \\
64\end{array}$ & $\begin{array}{l}32 \\
31\end{array}$ & $\begin{array}{l}70 \\
69\end{array}$ & $\begin{array}{l}28 \\
26\end{array}$ & $\begin{array}{l}76 \\
74\end{array}$ \\
\hline
\end{tabular}




\section{QUADRO 8}

Comparação dos limites de $\mathbf{f}(\mathrm{obs})$ para a distribuição binominal $\left(\frac{1}{2}+\frac{1}{2}\right)^{N}$ e o $\mathrm{x}^{2}$-teste com duas classes

\begin{tabular}{|c|c|c|c|c|c|c|c|c|}
\hline \multirow{3}{*}{ N. } & \multirow{3}{*}{ f.esp. } & \multirow{3}{*}{ Fórmula } & Preci & $105 \%$ & \multicolumn{2}{|c|}{ Precisão $1 \%$} & \multicolumn{2}{|c|}{ Prec. $0,1 \%$} \\
\hline & & & \multicolumn{6}{|c|}{ Limites de $f(e s p)}$. \\
\hline & & & Inf. & Sup. & Inf. & Sup. & Inf. & Sup. \\
\hline \multirow[t]{2}{*}{5} & 2,5 & Binomio & - & 一 & 一 & - & - & - \\
\hline & & $\mathrm{X}^{2}$-Total & - & - & - & - & - & \\
\hline \multirow[t]{2}{*}{6} & 3,0 & Binómio & $\overline{0}$ & 6 & - & 一 & - & - \\
\hline & 35 & & 0 & 6 & - & 二 & 二 & 二 \\
\hline 7 & 0,0 & X2-Total & $\begin{array}{l}0 \\
0\end{array}$ & 7 & 二 & 二 & 二 & I \\
\hline \multirow[t]{2}{*}{8} & 4,0 & Binômio & 0 & 8 & 0 & 8 & - & - \\
\hline & & $X^{2}$-Total & 1 & 7 & 0 & 8 & - & \\
\hline \multirow[t]{2}{*}{9} & 4,5 & Binômio & 1 & 8 & 0 & 9 & - & - \\
\hline & & $X^{2}$-Total & 1 & 8 & 0 & 9 & - & - \\
\hline 10 & 5,0 & $\begin{array}{l}\text { Binomio } \\
\text { X2-Total }^{2}\end{array}$ & $\begin{array}{l}1 \\
1\end{array}$ & $\begin{array}{l}9 \\
9\end{array}$ & $\begin{array}{l}0 \\
0\end{array}$ & $\begin{array}{l}10 \\
10\end{array}$ & 二 & - \\
\hline \multirow[t]{2}{*}{11} & 5,5 & Binómio & 1 & 10 & 0 & 11 & 0 & 11 \\
\hline & & & 2 & 9 & 1 & 10 & 0 & 11 \\
\hline \multirow[t]{2}{*}{12} & 6,0 & Binómio & 2 & 10 & 1 & 11 & $\mathbf{0}$ & 12 \\
\hline & & X2-Total & 2 & 10 & 1 & 11 & 0 & 12 \\
\hline \multirow[t]{2}{*}{14} & 7,0 & Binômio & 2 & 12 & 1 & 13 & 0 & \\
\hline & & & 3 & 11 & 2 & 12 & 0 & 14 \\
\hline 16 & 8,0 & Binômio & 3 & 13 & 2 & 14 & 1 & 15 \\
\hline & & & 4 & 12 & 2 & 14 & 1 & 15 \\
\hline 10 & 9,0 & Binomio & 4 & 14 & 3 & 15 & 1 & 17 \\
\hline 20 & 10,0 & Binomio & 5 & 15 & 3 & 17 & 2 & 18 \\
\hline & & $\mathbf{X}^{2}$-Total & 5 & 15 & 4 & 16 & $\overline{2}$ & 18 \\
\hline
\end{tabular}




\section{QUADRO 9}

Comparação dos limites de f(obs) para a distribuição binominal $\left(\frac{3}{4}+\frac{1}{4}\right)^{\mathrm{N}}$ e o $\mathbf{X}^{2}$-Teste com duas classes

\begin{tabular}{|c|c|c|c|c|c|c|c|c|}
\hline \multirow{3}{*}{ N. } & \multirow{3}{*}{ f.esp. } & \multirow{3}{*}{ Fórmula } & \multicolumn{2}{|c|}{ Precisão 5\% } & \multicolumn{2}{|c|}{ Precisão $1 \%$} & \multicolumn{2}{|c|}{ Prec. $0,1 \%$} \\
\hline & & & \multicolumn{6}{|c|}{ Limites de $f$ (esp.) } \\
\hline & & & Inf. & Sup. & Inf. & Sup. & Inf. & Sup. \\
\hline \multirow[t]{2}{*}{4} & 1,0 & Binómio & - & 3 & - & 4 & & \\
\hline & & $\mathrm{X}^{2}$-Total & - & 4 & - & 4 & $=$ & 二 \\
\hline \multirow[t]{2}{*}{6} & 1,5 & Binômio & - & 5 & - & 5 & - & 6 \\
\hline & & $\mathbf{X}^{2}$-'Total & - & 4 & - & $\mathbf{5}$ & - & 5 \\
\hline 8 & 2,0 & Binômio & $=$ & 6 & 一 & 6 & - & 7 \\
\hline \multirow[t]{2}{*}{10} & 2,5 & Binómio & - & $\begin{array}{l}5 \\
6\end{array}$ & - & $\begin{array}{l}0 \\
7\end{array}$ & - & 8 \\
\hline & & X2-Total & - & 6 & - & 7 & - & 8 \\
\hline \multirow[t]{2}{*}{12} & 3,0 & Binomio & - & 7 & - & 8 & - & 9 \\
\hline & & $\mathrm{X}^{2}$-Total & 0 & 6 & $\cdots$ & 7 & - & 8 \\
\hline \multirow[t]{2}{*}{14} & 3,5 & Binômio & 0 & 8 & - & 9 & - & 10 \\
\hline & & $\mathrm{X}^{2}$-Total & 0 & 7 & - & 8 & - & 9 \\
\hline \multirow[t]{2}{*}{16} & 4,0 & Binômio, & 0 & 9 & - & 10 & - & 11 \\
\hline & & $\mathrm{X}^{2}$-Total & 0 & 8 & - & 9 & & 10 \\
\hline \multirow[t]{2}{*}{18} & 4,5 & Binômio. & D & 9 & - & 11 & - & 12 \\
\hline & & X2-Total & 0 & 9 & - & 10 & - & 11 \\
\hline \multirow{2}{*}{20} & 5,0 & Binômio. & 1 & 10 & 0 & 11 & - & 13 \\
\hline & & X2-Total & 1 & 9 & 0 & 10 & - & 12 \\
\hline \multirow[t]{2}{*}{24} & 6 & Binômio & 1 & 11 & 0 & 13 & - & 15 \\
\hline & & $\mathbf{X}^{2}$-Total & 1 & 11 & 0 & 12 & - & 13 \\
\hline \multirow[t]{2}{*}{28} & 7 & Binômio. & 2 & 13 & 1 & 14 & 0 & 16 \\
\hline & & $X^{2}$-Total & 2 & 12 & 1 & 13 & 1 & 15 \\
\hline \multirow{2}{*}{32} & 8 & Binomio & 3 & 14 & $\overline{1}$ & 16 & 0 & 18 \\
\hline & & $\mathbf{X}^{2}$-Tot & 3 & 13 & 1 & 15 & 0 & 16 \\
\hline \multirow[t]{2}{*}{36} & 9 & Binómio & 3 & 15 & 2 & 17 & 1 & 19 \\
\hline & & $\mathrm{X}^{2}$-Total & 3 & 15 & $\overline{2}$ & 16 & 0 & 18 \\
\hline \multirow[t]{2}{*}{40} & 10 & Binômio & 4 & 17 & $\overline{3}$ & 18 & 1 & 21 \\
\hline & & $\mathrm{X}^{2}$-Total & 4 & 16 & 2 & 18 & 0 & 20 \\
\hline
\end{tabular}




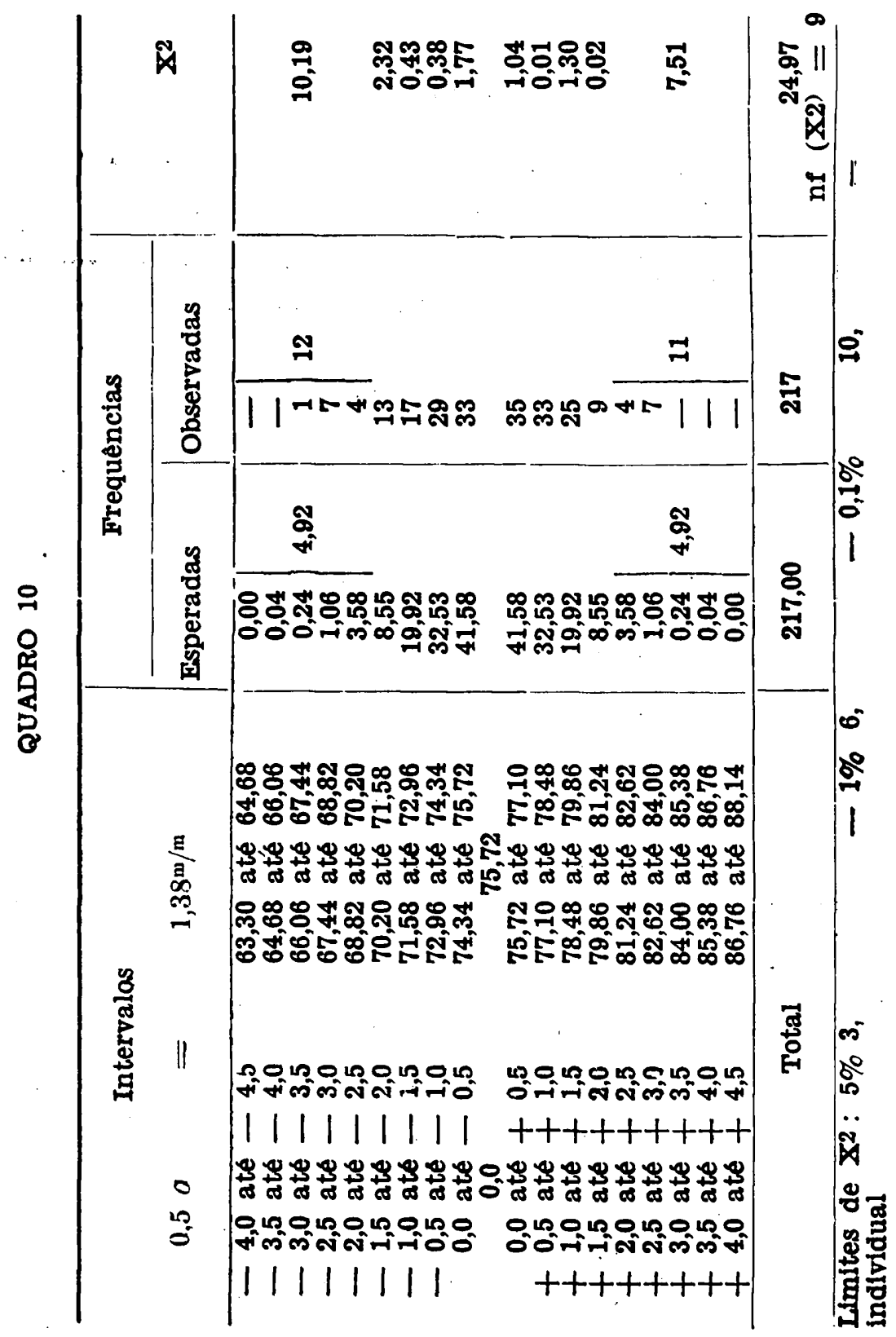




\section{TABOA I}

Valores extremos da frequência observada na dístribuição de Poisson que não devem mais ser encontrados

\begin{tabular}{|c|c|c|c|c|c|c|}
\hline \multirow{3}{*}{ f.esp. } & \multicolumn{2}{|c|}{ Precisão 5\% } & \multicolumn{2}{|c|}{ Precisão $1 \%$} & \multicolumn{2}{|c|}{ Precisão $0,1 \%$} \\
\hline & \multicolumn{6}{|c|}{ Limites de $\mathbf{f}$ (obs) } \\
\hline & Inf. & Sup. & Inf. & Sup. & Inf. & Sup. \\
\hline $\begin{array}{c}0,001 \\
0,005 \\
0,01 \\
0,05 \\
0,1 \\
0,2 \\
0,3 \\
0,4 \\
0,5 \\
0,6 \\
0,7 \\
0,8 \\
0,9 \\
1,0 \\
1,5 \\
2,0 \\
2,5 \\
3,0 \\
3,5 \\
4,0 \\
4,5 \\
5,0 \\
6,0 \\
7,0 \\
8,0 \\
9,0 \\
10,0 \\
11,0 \\
12,0 \\
13,0 \\
14,0 \\
15,0\end{array}$ & 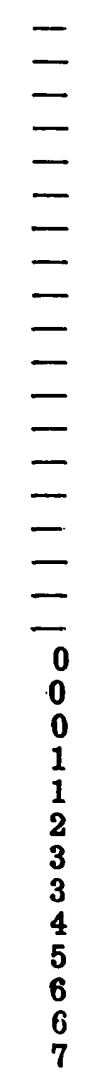 & $\begin{array}{r}1 \\
1 \\
1 \\
2 \\
1 \\
2 \\
3 \\
3 \\
3 \\
3 \\
4 \\
4 \\
4 \\
4 \\
5 \\
6 \\
7 \\
8 \\
9 \\
9 \\
10 \\
11 \\
12 \\
14 \\
15 \\
16 \\
18 \\
19 \\
20 \\
22 \\
23 \\
24\end{array}$ & $\begin{array}{l}= \\
= \\
= \\
= \\
= \\
= \\
= \\
= \\
= \\
= \\
= \\
= \\
= \\
= \\
0 \\
0 \\
1 \\
1 \\
2 \\
3 \\
4 \\
4 \\
5\end{array}$ & $\begin{array}{r}1 \\
1 \\
2 \\
2 \\
2 \\
3 \\
3 \\
4 \\
4 \\
4 \\
5 \\
5 \\
5 \\
5 \\
6 \\
7 \\
8 \\
9 \\
10 \\
11 \\
12 \\
13 \\
14 \\
16 \\
17 \\
19 \\
20 \\
21 \\
23 \\
24 \\
26 \\
27\end{array}$ & $\begin{array}{l}= \\
= \\
= \\
= \\
= \\
= \\
= \\
= \\
= \\
= \\
= \\
= \\
= \\
= \\
= \\
\bar{Z} \\
0 \\
0 \\
1 \\
1 \\
1 \\
2 \\
3 \\
3\end{array}$ & $\begin{array}{r}2 \\
2 \\
2 \\
3 \\
3 \\
4 \\
4 \\
5 \\
5 \\
5 \\
6 \\
6 \\
6 \\
7 \\
8 \\
9 \\
10 \\
11 \\
12 \\
13 \\
14 \\
15 \\
17 \\
18 \\
20 \\
21 \\
23 \\
24 \\
26 \\
27 \\
29 \\
30\end{array}$ \\
\hline
\end{tabular}


TABOA II

Valores extremos da frequência observada na distribuição binominal que não devem mais ser encontrados

\begin{tabular}{|c|c|c|c|c|c|c|c|}
\hline \multirow{3}{*}{ Tot. } & \multirow{3}{*}{ f.esp. } & \multicolumn{2}{|c|}{ Precisão $\quad 5 \%$} & \multicolumn{2}{|c|}{ Precisão $1 \%$} & \multicolumn{2}{|c|}{ Precisáo 0,1\% } \\
\hline & & \multicolumn{6}{|c|}{ Limites de $f$ (obs) } \\
\hline & & Inf. & Sup. & Inf. & Sup. & Inf. & Sup. \\
\hline \multirow[b]{2}{*}{$\begin{array}{r}5 \\
6 \\
7 \\
8 \\
9 \\
10 \\
11 \\
12 \\
14 \\
16 \\
18 \\
20\end{array}$} & \multirow[b]{2}{*}{$\begin{array}{r}2,5 \\
3,0 \\
3,5 \\
4,0 \\
4,5 \\
5,0 \\
5,5 \\
6,0 \\
7,0 \\
8,0 \\
9,0 \\
10,0\end{array}$} & \multicolumn{4}{|c|}{ Binómio $\frac{(1}{2}+\frac{1)^{n}}{2}$} & & \\
\hline & & $\begin{array}{l}- \\
0 \\
0 \\
1 \\
1 \\
1 \\
2 \\
2 \\
3 \\
4 \\
5\end{array}$ & $\begin{array}{r}-6 \\
7 \\
8 \\
8 \\
9 \\
10 \\
10 \\
12 \\
13 \\
14 \\
15\end{array}$ & $\begin{array}{c}- \\
- \\
0 \\
0 \\
0 \\
0 \\
1 \\
1 \\
2 \\
3 \\
3\end{array}$ & $\begin{array}{r}- \\
- \\
8 \\
9 \\
10 \\
11 \\
11 \\
13 \\
14 \\
15 \\
17\end{array}$ & $\begin{array}{l}\text { - } \\
\text { - } \\
\text { - } \\
\text { D } \\
0 \\
0 \\
1 \\
1 \\
2\end{array}$ & $\begin{array}{l}- \\
- \\
- \\
- \\
- \\
11 \\
12 \\
14 \\
15 \\
17 \\
18\end{array}$ \\
\hline \multirow[b]{2}{*}{$\begin{array}{r}2 \\
4 \\
6 \\
8 \\
10 \\
12 \\
14 \\
16 \\
18 \\
20 \\
24 \\
28 \\
32 \\
36 \\
49\end{array}$} & \multirow[b]{2}{*}{$\begin{array}{r}0,5 \\
1,0 \\
1,5 \\
\mathbf{2}, 0 \\
2,5 \\
3,0 \\
3,5 \\
4,0 \\
4,5 \\
5,0 \\
6,0 \\
7,0 \\
8,0 \\
9,0 \\
10,0\end{array}$} & \multicolumn{4}{|c|}{ Binômio $\frac{(3}{4}+\frac{1)^{n}}{4}$} & & \\
\hline & & $\begin{array}{l}- \\
- \\
- \\
- \\
- \\
0 \\
0 \\
0 \\
1 \\
1 \\
2 \\
3 \\
3 \\
4\end{array}$ & $\begin{array}{r}3 \\
5 \\
6 \\
6 \\
7 \\
8 \\
9 \\
9 \\
10 \\
11 \\
13 \\
14 \\
15 \\
17 \\
\end{array}$ & $\begin{array}{l}- \\
Z \\
\bar{Z} \\
\bar{Z} \\
\bar{Z} \\
\bar{Z} \\
\overline{0} \\
0 \\
1 \\
1 \\
2 \\
3\end{array}$ & $\begin{array}{r}4 \\
5 \\
6 \\
7 \\
8 \\
9 \\
10 \\
11 \\
11 \\
13 \\
14 \\
16 \\
17 \\
18\end{array}$ & $\begin{array}{l}- \\
- \\
= \\
= \\
\bar{Z} \\
\overline{-} \\
\overline{-} \\
\overline{0} \\
0 \\
1 \\
1\end{array}$ & $\begin{array}{r}- \\
6 \\
7 \\
8 \\
9 \\
10 \\
11 \\
12 \\
13 \\
15 \\
16 \\
18 \\
19 \\
21\end{array}$ \\
\hline
\end{tabular}


TABOA III

Limites de $\mathbf{X}^{2}$

\begin{tabular}{|c|c|c|c|c|}
\hline$n\left(X^{2}\right)$ & $0,1 \%$ & $1 \%$ & $5 \%$ & $\mathrm{n}\left(\mathrm{X}^{2}\right)$ \\
\hline $\begin{array}{r}1 \\
2 \\
3 \\
4 \\
5 \\
6 \\
7 \\
8 \\
9 \\
10 \\
11 \\
12 \\
13 \\
14 \\
15 \\
16 \\
17 \\
18 \\
19 \\
20 \\
21 \\
22 \\
23 \\
24 \\
25 \\
26 \\
27 \\
28 \\
29 \\
30\end{array}$ & $\begin{array}{l}10,83 \\
13,82 \\
16,27 \\
18,47 \\
20,52 \\
22,46 \\
24,32 \\
26,13 \\
27,88 \\
29,59 \\
31,26 \\
32,91 \\
34,53 \\
36,12 \\
37,70 \\
39,25 \\
40,79 \\
42,31 \\
43,82 \\
45,32 \\
46,80 \\
48,27 \\
49,73 \\
51,18 \\
52,62 \\
54,05 \\
55,48 \\
56,89 \\
58,30 \\
59,70\end{array}$ & $\begin{array}{r}6,66 \\
9,21 \\
11,35 \\
13,28 \\
15,09 \\
16,81 \\
18,48 \\
20,09 \\
21,67 \\
23,21 \\
24,73 \\
26,22 \\
27,69 \\
29,14 \\
30,58 \\
32,00 \\
33,41 \\
34,81 \\
36,19 \\
37,57 \\
38,93 \\
40,29 \\
41,64 \\
42,98 \\
44,31 \\
45,64 \\
46,96 \\
48,28 \\
49,59 \\
50,89\end{array}$ & $\begin{array}{r}3,84 \\
5,99 \\
7,82 \\
9,49 \\
11,07 \\
12,59 \\
14,07 \\
15,51 \\
16,92 \\
18,31 \\
19,68 \\
21,03 \\
22,36 \\
23,69 \\
25,00 \\
26,30 \\
27,59 \\
28,87 \\
30,14 \\
31,41 \\
32,67 \\
33,92 \\
35,17 \\
36,42 \\
37,65 \\
38,89 \\
40,11 \\
41,34 \\
42,56 \\
43,77\end{array}$ & $\begin{array}{r}1 \\
2 \\
3 \\
4 \\
5 \\
6 \\
7 \\
8 \\
9 \\
10 \\
11 \\
12 \\
13 \\
14 \\
15 \\
16 \\
17 \\
18 \\
19 \\
20 \\
21 \\
22 \\
23 \\
24 \\
25 \\
26 \\
27 \\
28 \\
29 \\
30\end{array}$ \\
\hline
\end{tabular}


